\title{
Techniques developed for the ATLAS Thin Gap Chambers mass production in Japan
}

\author{
S.Tanaka ${ }^{1}$,H.Ohshita ${ }^{2}$,K.Ishii ${ }^{3}$,H.Iwasaki ${ }^{1}$,Y.Arataki ${ }^{3}$,T.Bando ${ }^{4}$, Y.Homma ${ }^{5}$,M.Ishino ${ }^{4}$,T.Kondo ${ }^{1}$, \\ T.Kobayashi ${ }^{4}$,H.Kurashige ${ }^{3}$,G.Mikenberg ${ }^{6}$,Y.Miyazaki ${ }^{2}$, Y.Nakagawa ${ }^{2},{ }^{2}$ H.Nanjo ${ }^{4}$, M.Ikeno ${ }^{1}$,

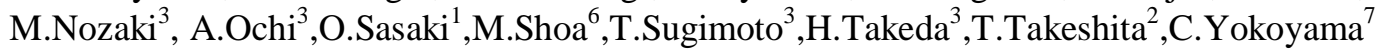 \\ ${ }^{1}$ KEK,Tsukuba, Japan \\ ${ }^{2}$ Faculty of Science, Shinshu University, Matsumoto, Japan \\ ${ }^{3}$ Graduate school of Science and Technology, Kobe University, Kobe, Japan \\ ${ }^{4}$ International Center for Elementary Particle Physics (ICEPP), University of Tokyo, Tokyo, Japan \\ ${ }^{5}$ Faculty of Engineering, Kobe University, Kobe, Japan \\ ${ }^{6}$ Weizmann Institute of Science, Rehovot, Israel \\ ${ }^{7}$ Institute for Cosmic Ray Research (ICRR), University of Tokyo, Kashiwa, Japan
}

\begin{abstract}
The Thin Gap Chambers (TGCs) are used for the muon trigger system in the end-cap regions of the ATLAS detector. The TGC mass production phase at High Energy Accelerator Research Organization (KEK) started in January 2001. As the anode-cathode distance is small, $1.4 \mathrm{~mm}$, chamber flatness is essential to achieve a uniform gas gain over the chamber. In order to perform a stable production with high quality we developed a chamber closing system. When we glue two half-chambers together, we sandwich them between a granite table and an aluminum honeycomb panel to keep the chamber flat from both sides. By using silk screens, we control the quantity of epoxy adhesive that affects the chamber thickness. Due to these developments, we can achieve the flatness of less than $100 \mu \mathrm{m}$. Uniformity of detection efficiency of the TGC is measured with a cosmic-ray test bench at Kobe University. So far we have tested 300 TGCs. Position dependence of the efficiency is measured with a granularity of $5 \mathrm{~mm}-\mathrm{by}-5 \mathrm{~mm}$. The average efficiency over the tested chambers is achieved to be $99 \%$ excluding the wire supports and spacers.
\end{abstract}

Index Terms- Gas detectors, Proportional counters, Triggering

\section{INTRODUCTION}

The Thin Gap Chambers (TGCs) are used for the muon trigger system in the end-cap regions of the ATLAS detector [1]-[3]. The TGC is characterized by fast signal response (99\% of the output signals are within $25 \mathrm{~ns}$ ) for charged particles [4], [5]. This characteristic suits the muon trigger detector of the Large Hadron Collider (LHC), which is required to identify the bunch crossing at $40 \mathrm{MHz}$. Each TGC has a trapezoidal shape, whose dimensions depend on its location. A typical size is $1.3 \mathrm{~m}$ (longer base) $\times 1.3 \mathrm{~m}$ (height).
As the anode-cathode distance is small, $1.4 \mathrm{~mm}$ (Fig. 1), chamber flatness is essential to achieve a uniform gas gain over the chamber. The parameters of the TGC structure and the operation conditions are summarized in Table I. The TGC is designed to provide a fast signal response for charged particles. The diameter of the wire is $50 \mu \mathrm{m}$ in order to give a wide range and high electric field. The surface of glass-epoxy laminate (FR-4) in the gas volume is coated with graphite, which surves as the cathode plane. Its nominal surface resistivity is $1 \mathrm{M} \Omega /$ squre m. Pickup read-out strips made of copper foil run perpendicularly to the wire, to give the orthogonal coordinate, on the surface of FR-4 opposite to the gas volume.

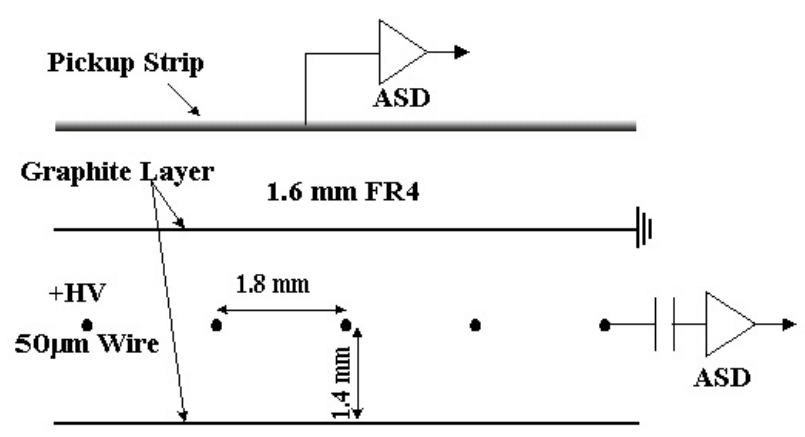

Fig. 1. Schematic view of a cross section of the TGC. ASD stands for an amplifier-shaper-discriminator readout channel.

ATLAS TGC chambers are produced in parallel in Japan, Israel and China. Basic performances of materials used in gas volumes were studied at KEK (Japan) in small prototype chambers [6], [7]. Production procedures of real-size TGCs 
were developed from March 1998 to the end of 2000. The TGC series production started in January 2001. A production of about 1100 TGCs is to be completed in Japan by the middle of 2004. In order to meet this schedule the KEK production facility was designed to produce two TGCs per day with about twelve workers and three physicists as supervisors.

TABLE I

CHARACTERISTICS OF THIN GAP CHAMBERS

\begin{tabular}{ll}
\hline \hline Parameter & Nominal value \\
\hline Gas gap & $2.8 \mathrm{~mm}$ \\
Anode wire pitch & $1.8 \mathrm{~mm}$ \\
Wire diameter & $50 \mu \mathrm{m}$ \\
Wire potential & $2900 \mathrm{~V}$ \\
Gas mixture & $\mathrm{CO}_{2}+n$-pentane $(55: 45)$ \\
\hline \hline
\end{tabular}

\section{Production Procedures}

In order to avoid the sag of the anode wire there rows wire supports in the gas volume. In addition, there are zigzag row of small buttons between the supports to keep the anode-cathode gap constant. A TGC with one gas gap is called "singlet" and two or three singlets glued together with honeycomb-panel inbetween are called "doublet" or "triplet", respectively [1]. The TGC production procedures can be roughly divided into nine stages: checking the quality of the materials, graphite spraying, FR-4 frame gluing, wire winding, singlet TGC closing, sealing around the singlet, doublets or triplets modules production, mounting read-out boards, assembling $\mathrm{CO}_{2}$-gas channels around the module. All the procedures except the graphite spraying are performed in parallel. In order to reach the desired high quality the TGC production line is required to keep a precise anode wires spacing $(1.8 \mathrm{~mm})$ for a uniform time response, flatness of both anode and cathode planes to obtain a uniform gas gain and cleanness of the detection gas volume to avoid discharges. The main part of the TGC is made of commercially available FR-4 boards.

\section{A. Wire winding}

Since more than 700,000 wires are to be soldered in the whole production at KEK, precise and reliable wire winding and soldering technique had to be established. The $50 \mu \mathrm{m}$ anode wires are strung by using an automatic winding machine, which can control the wire pitch with precision of 1 micron and the wire tension within $3 \%$ error of designed value, 350 gw. The automatic winding machine consists of a linear actuator and a rotating table. Two cathode frames are hold on both sides of the table by small fixing pieces around the frame and twelve suction pads at the central part. The linear actuator moves half wire pitch every half turn of the rotating table. The high accuracy in the position of the linear actuator is achieved by a feed back control of the servomotor with a linear encoder.
To solder the anode wire we use tin-zinc (80/20) solder. It can hold the wire against higher tension than normally used tin-lead based solder. Since residual of the solder flux causes the ion-migration and weaken the strength by corrosion, we choose a water-soluble flux. We can clean the soldered region with dematerialized water applying supersonic wave before washing the cathode planes.

\section{B. Washing frames}

During the wire soldering, sometimes nebulized flux drops adhere on the cathode plane. Its remaining ion contaminant of the flux might cause discharge in the operation. In order to clean the surface of the cathode plane, we use an automatic washing machine before closing the singlets. The machine showers dematerialized water mist on the whole cathode frame. After the shower, a nozzle mounted on a linear actuator scans the frames and sprays compressed air in order to dry them.

\section{Closing singlet TGC}

After the wire winding stage, the wire tension causes the frame to arch with a few $\mathrm{cm}$ gaps between the wires to the cathode plane. We adopted a combination of vacuum-press and a suction plate technique for gluing to make a singlet TGC as it can apply uniform force on both sides of the TGC.

Fig. 2. Schematic view of a setup of closing singlet TGC. The lower frame with wires is sucked to the granite table with $-40 \mathrm{kPa}$. The upper TGC is sucked to the aluminum honeycomb. Two frames are pressed by the atmospheric pressure with $-10 \mathrm{kPa}$. The pressure inside the silicon rubber sheet $(-10 \mathrm{kPa})$ is maintained by a pressure controller with supplying air.

As shown in Fig.2, the frame with wires places on the granite table and another frame without wires is hold under an aluminum honeycomb plate (AL-plate). On the surface of the granite table, a polypropylene sheet (PP sheet) with 30 mesh per inch is attached. A 0.5 -mm-thick silicon rubber strip, which its surface is treated with isopropyl alcohol to make the surface sticky, is attached with double-coated tape around the PP sheet for sealing. There are four suction holes for sucking the frame with wire onto it. Since the thicknesses of the PP sheet and silicon rubber are the same and the homogeneous suction force is applied, the frame can be kept flat. Similarly, the frame without wires is sucked onto the AL-plate. To suck the frames, regulated negative pressure $(-40 \mathrm{kPa})$ is applied between the granite table and the frame with wires, as well as between the AL-plate and the frame without wires. 
The two frames with the AL-plate are covered with a silicon rubber sheet. Inside the volume surrounded by the granite table and the rubber sheet is then decompressed (-10 kPa Gauge: Pressure1) to press the two frames uniformly. Since the seal of the 0.5 -mm-thick silicon rubber strip is not perfect, Pressure 1 would become lower and lower and eventually reach the same level as Pressure2 $(-40 \mathrm{kPa})$. In order to avoid such an over pressure on the frames, Pressure1 is maintained by supplying clean air controlled with a pressure sensor and gas ballast.

As for the technique of applying adhesives, a screen-printing method is adopted. We use a polyarylate mesh screen (145 meshes per inch) to apply the epoxy to the wire-supports and frames. The thickness and the width of the adhesive can be uniformly adjusted. The screen is tough enough for multi-use and the residual resin can be easily removed by water. The resulting surface distortions of the singlet TGCs are shown in Fig.3. Almost all the singles reach a flatness of less than 100 $\mu \mathrm{m}$.

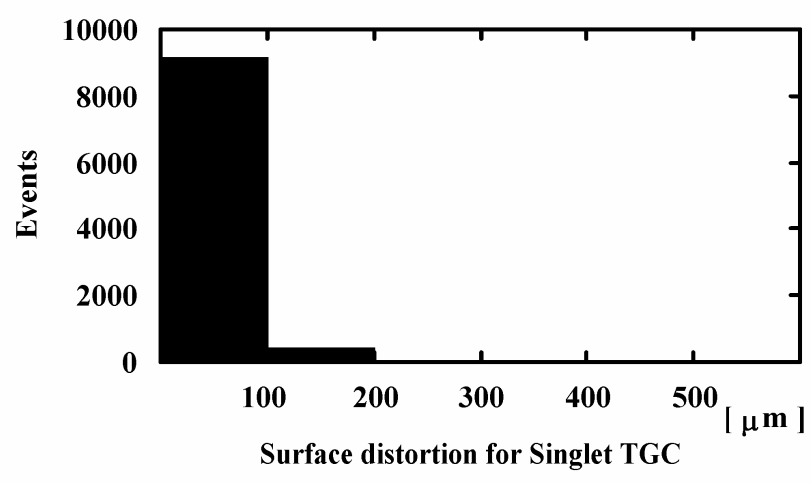

Fig. 3. Surface flatness distribution of the singlet TGC.

\section{Making modules}

Fig. 4. Schematic view of a setup of the doublet gluing process. The upper TGC is sucked to the aluminum honeycomb with $-40 \mathrm{kPa}$. The lower TGC is sucked to the granite table. Two TGCs are pressed by the atmospheric pressure with $-10 \mathrm{kPa}$.

A similar pneumatic control system is developed for assembling the doublet (or triplet). Fig. 4 schematically shows the doublet assembly procedure. Two singlet TGCs are kept flat by being sucked (-40 $\mathrm{kPa}$ : Pressure2) to either a granite table (bottom) or an AL-plate (top). A 20-mm-thick paper honeycomb and module support frames are sandwiched by these two singlet TGCs and glued together at one time. To apply a uniform force onto the TGCs, all the subunits of the doublet are covered with a silicon rubber sheet inside of which is decompressed to $-10 \mathrm{kPa}$ (Pressure1) with a pressure regulator. The gas volumes of the singlet TGCs are slightly over-pressured $(150 \mathrm{~Pa})$ with clean air from the TGC gas inlets as counter force against the force on the rubber sheet.

\section{QUALITY ASSURANCE}

Since the TGCs are assembled with adhesive, once the adhesive is cured it is not possible to return to a pervious stage without damaging the chamber. Therefore, it is important to check the quality at each assembly stage before moving to the next one. We have adopted the following quality checks: a measurement of the resistivity of the graphite surface, high voltage tests (HV tests), a pulse test to check the connection of signal routing, and a signal response test with a radioactive source.

\section{A. Cathode surface resistivity control}

The surface resistivity of the graphite sprayed should be approximately $1 \mathrm{M} \Omega /$ square in order to reduce cross talk between strip channels while still avoiding voltage drop [1]. To realize the uniform surface resistivity, we use an automatic sprayer composed of a two-dimensional linear actuator and a spray gun with pneumatic control. The sprayer can unformly paint the whole cathode plane with a thickness of about $10 \mu \mathrm{m}$. The surface resistivity is measured at about 66 sampling points on a plane. If they are smaller than $0.5 \mathrm{M} \Omega$ /square, then the plane is cleaned up and is sprayed again. If it exceeds 1.5 $\mathrm{M} \Omega /$ squre, the area is polished with soft paper until it reaches $1.5 \mathrm{M} \Omega /$ squre.

\section{B. High voltage tests}

We apply high voltage at three stages and check leak current in the course of the whole assembly: before closing the singlet TGC, after the closure, and the assembly of adaptor boards which bring signals from wires or strips to the ASDs. The purpose of the HV test is to verify that there is no serious problem up to this stage: no broken or slack wires, and no fine dusts or chemicals inside the gas volume which would cause problems later on. The acceptance criterion of all these tests is that the chamber current should be less than $100 \mathrm{nA}$ at the applied voltage of $2.8 \mathrm{kV}$ with $\mathrm{CO}_{2}$ gas.

Before closing singlet the HV test is a particulary important feature in the production. The setup is the same as closing singlet (Fig. 2) except for feeding $\mathrm{CO}_{2}$ gas into the silicon rubber. If we find steady high leak current or some discharges, 
we reopen the frames and wash the inside of the frames. Then we do the HV test again. We repeat this procedure until the leak current satisfies the criterion. Since the pressure in the gas volume is $-10 \mathrm{kPa}$, the $\mathrm{HV}$ of $2.8 \mathrm{kV}$ corresponds to $3.1 \mathrm{kV}$ at the standard atomospheric pressure. Fig.5 shows a distribution of the leak current measured at the first cycle of the tests. From this distribution, one can see majority of the singlet TGCs pass this test at its first attempt.

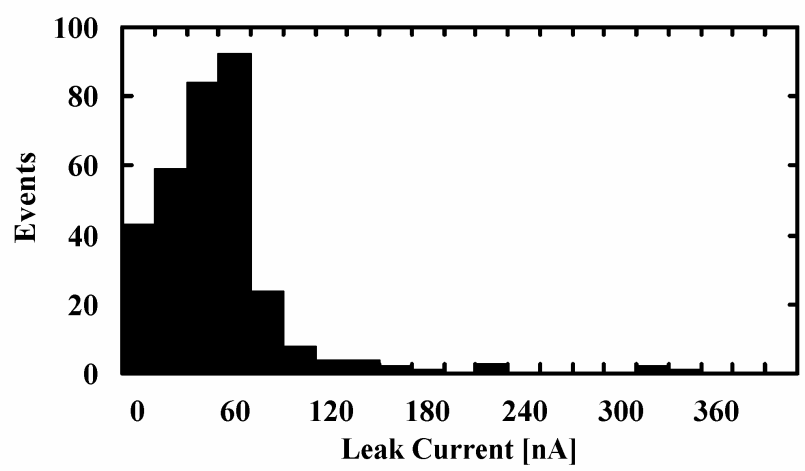

Fig. 5. Leak current distribution at $2.8 \mathrm{kV}$ with $\mathrm{CO}_{2}$ gas before closing singlet TGC.

\section{Pulse test}

After the doublet or triplet assembly, we attach the so called adaptor boards which brings signals from the wires or strips to the ASDs. After the attachment, we check the correctness of the connections and verify there is no shortage between the adjecent signal lines or to the ground. A pulse test is adopted for this purpose. A rectangle pulse is applied to the TGC HV supply line. The output pulse through the RC-CR circuit from each channel is recorded with a digital oscilloscope. Fig. 6 shows a shematical diagram of the electrical circuit of TGC [8]. If a channel is shorted to the adjacent one, its output pulse becomes approximately twice larger than the nominal; if its signal path is broken or is shorted to the ground, no output pulse is observed.

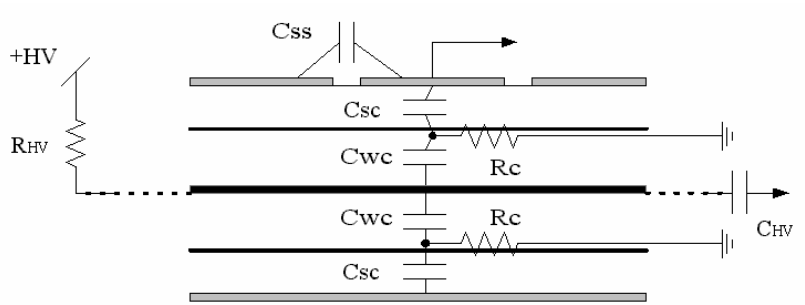

Fig. 6. The schematic view of the electrical circuit inside the TGC. $\mathrm{R}_{\mathrm{HV}}$ is the input resistor, $\mathrm{R}_{\mathrm{C}}$ the surface resistivity of the graphite paint, $\mathrm{C}_{\mathrm{SS}}$ the capacitance of adjoining pickup strips, $\mathrm{C}_{\mathrm{WC}}$ the capacitance between the anode wire and the cathode plane, $\mathrm{C}_{\mathrm{SC}}$ the capacitance between the cathode plane and the pickup strip and $\mathrm{C}_{\mathrm{HV}}$ the coupling capacitor for the anode wire read-out.

The results are shown in Fig. 7. The muximun fluctuations of the pulse height are about $\pm 6 \%$ for the wire and $\pm 12 \%$ for the strip. They are both much less than two. Using the SPICE program [1] we simulated the pulse height dependence on the the cathode surface resistivity and the anode-cathode distance. The results are given in Table II. It shows a strip deviation could be on the order of $10 \%$ due to the deviation in surface resistivity. In addition, the quoted errors of the coupling capacitor for the wire $\left(\mathrm{C}_{\mathrm{HV}}\right)$ and the decoupling resistor at the $\mathrm{HV}$ input $\left(\mathrm{R}_{\mathrm{HV}}\right)$ are $10 \%$ and $5 \%$, respectively. These errors could cause pulse height fluctuations of up to $3 \%$ for the resistor or $6 \%$ for the capacitor. The distribution of the signal output pulse height are therefore consistent with these errors and the surface resistivity criterion.
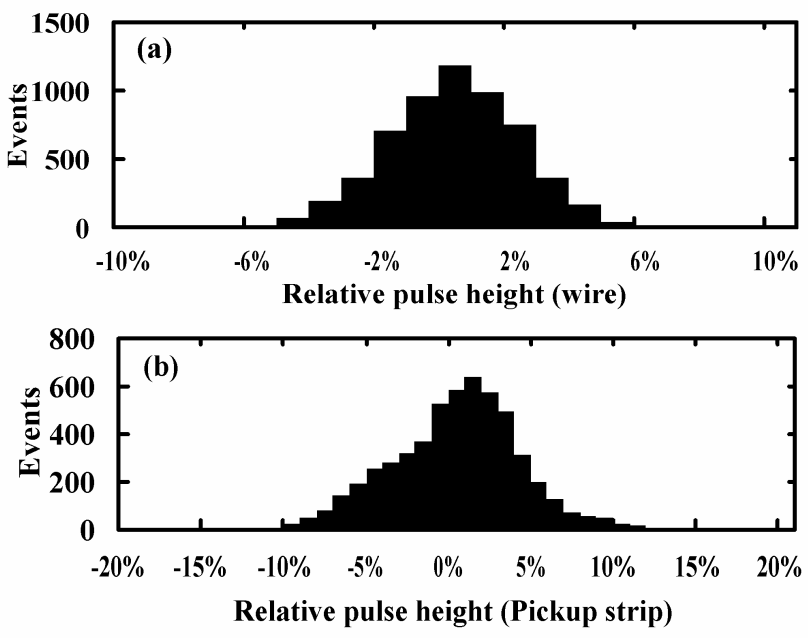

Fig. 7. The relative pulse height distribution for anode wire and pickup strip read-out on the pulse test.

TABLE II

PULSE HEIGHT DEPENDENCE ON THE CATHODE SURFACE RESISTIVITY AND THE ANODE-CATHODE DISTANCE. ESTIMATED WITH SPICE SIMULATION.

\begin{tabular}{llrrrr}
\hline \hline & Gas Gap & $0.5 \mathrm{M} \Omega$ & $1.0 \mathrm{M} \Omega$ & $2.0 \mathrm{M} \Omega$ & $3.0 \mathrm{M} \Omega$ \\
\hline \multirow{3}{*}{ Wire } & $1.2 \mathrm{~mm}$ & -3 & -1.8 & 1.2 & 4.2 \\
& $1.4 \mathrm{~mm}$ & -1.2 & 0.0 & 2.4 & 4.8 \\
& $1.6 \mathrm{~mm}$ & 0.6 & 1.8 & 3.6 & 6.0 \\
\hline \multirow{3}{*}{ Strip } & $1.2 \mathrm{~mm}$ & -10.4 & 4.5 & 18.0 & 23.6 \\
& $1.4 \mathrm{~mm}$ & -14.1 & 0.0 & 12.4 & 19.1 \\
& $1.6 \mathrm{~mm}$ & -16.4 & -2.1 & 10.1 & 15.7 \\
\hline \hline [UNIT: $\%$ ]
\end{tabular}

\section{D. $\beta$-ray test}

After the pulse test, we check the basic functionality of the TGCs with using a $\beta$-ray radioactive source. The chambers are operated with $\mathrm{CO}_{2}$ at $2.8 \mathrm{kV}$. We investigate the signal output rate searching for noisy channels. We also check for oscillations caused by grounding problems.

\section{DETECTOR PERformanCE}

The last quality assurance stage is a measurement of the detection efficiency uniformity of all the produced TGC in a 
dedicated test bench at Kobe University. We set the operating point of the TGC just above the shoulder of the HV curve to emphasize the non-uniformity, if it exists, while keeping high detection efficiency for the normal TGCs. The Kobe cosmic ray test bench can test 24 singlets at a time. Up to now 100 triplets (or equivalently TGC 300 singlets) have been tested.

Fig. 8. Example of detection efficiency maps. White dots show better than $99 \%$, deep gray less than $60 \%$. (a) A Typical chamber showing good uniformity. Five vertical lines correspond to the wire supports. Small zigzag points in-between correspond to the button spacers. (b) Bad chamber having several inefficient regions.

Position dependence of the efficiency was measured with a granularity of $5 \mathrm{~mm}$-by-5mm. It takes about two weeks for datataking to get one full map. Fig. 8 shows two examples of the detection efficiency maps. Fig. 8(a) is a typical example. Excluding the wire-supports and spacers, the average efficiency is achieved to be better than $99 \%$. Fig. 8(b) is a bad example of a chamber with some inefficient regions. There are seven chambers showing similar non-uniformity in total. These chambers were produced in relatively early period of the series production. The reason could be a gluing-off or applying thicker glue during the triplet assembly. This could be a result of failure in the viscosity control of the epoxy adhesive.

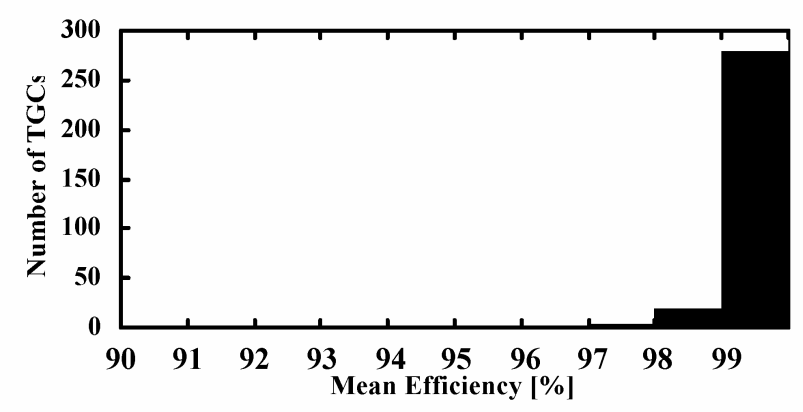

Fig. 9. Mean detection efficiency distribution for each chamber

We summarize the mean detection efficiencies in Fig. 9. There are 8 TGCs having less than $98 \%$ detection efficiency. Excluding the above-mentioned 6 TGCs with the epoxy problem. There are only two additional TGCs which show a poor quality than the average. There is a correlation between the non-uniformity or lower efficiency and the flatness of the triplet, but little correlation with the flatness of the singlet. This indicates that the flatness of singlet might have been damaged during the doublet (or triplet) assembly. We continue to study the problems and trying to reduce the number of poor performance TGCs. All the other produced TGCs demonstrated a good uniformity with high detection efficiency.

\section{REFERENCES}

[1] ATLAS Muon Technical Design Report, CERN/LHCC/97-22. ATLAS TDR, May 1997.

[2] K. Nagai. (1996, Dec.). Thin gap chambers in ATLAS. Nucl. Instrum. Methods. vol. A384, pp. 219-221.

[3] A. Rimoldi. (1998, May). The ATLAS muon trigger chamber system. Nucl. Instrum. Methods. vol. A409, pp. 669-674.

[4] D. Lazic, N. Lupu, A. Mincer, Y. Rozen, S. Tarem and A. Breskin et al. (1998, Jun.). Drift velocity in n-pentane mixtures and its influence on timing properties of thin gap chambers. Nucl. Instrum. Methods. vol. A410, pp. 159-165.

[5] Y. Arai, M. Asai, E. Barberio, T. Emura, J. Goldberg and K. Homma et al. (1995, Dec.). Timing optimization of thin gap chambers for the use in the ATLAS muon end-cap trigger. Nucl. Instrum. Methods. vol. A367, pp. 398-401.

[6] H. Fukui, M. Yoshida, Y. Miyazaki, Y. Fukatsu, Y. Hasegawa and T. Hosoda et al. (1998, Dec.). Studies on ageing effects and rate dependence of Thin Gap Chambers. Nucl. Instrum. Methods. vol. A419, pp. 497-502.

[7] A. I. Mincer, S. Dado, J. J. Goldberg, Y. Gernitzky, D. Lazic and N. Z. Lupu et al. (2000, Jan.). Charge production in thin gap multi-wire chambers. Nucl. Instrum. Methods. vol. A439, pp. 147-157.

[8] O. Sasaki and M.Yoshida. (1999, Dec.). ASD IC for the Thin Gap Chambers in the LHC Atlas Experiment. IEEE Trans. Nucl. Sci. vol. 46, pp. $1871-1975$ 